Musées, Patrimoine et Culture scientifiques et techniques

\title{
Le Réseau des musées de Normandie. Nouveau territoire, nouvelles coopérations?
}

\section{Élise Fin-Osinski et Margot Frénéa}

\section{OpenEdition \\ Journals}

Édition électronique

URL : http://journals.openedition.org/ocim/3246

DOI : 10.4000/ocim.3246

ISSN : 2108-646X

Éditeur

OCIM

Édition imprimée

Date de publication : 1 septembre 2019

Pagination : 26-35

ISSN : 0994-1908

Référence électronique

Élise Fin-Osinski et Margot Frénéa, « Le Réseau des musées de Normandie. Nouveau territoire, nouvelles coopérations? ", La Lettre de l'OCIM [En ligne], 185 | 2019, mis en ligne le 01 septembre 2020, consulté le 25 janvier 2021. URL : http://journals.openedition.org/ocim/3246 ; DOl : https:// doi.org/10.4000/ocim.3246

Ce document a été généré automatiquement le 25 janvier 2021.

Tous droits réservés 


\section{Le Réseau des musées de Normandie. Nouveau territoire, nouvelles coopérations?}

Élise Fin-Osinski et Margot Frénéa

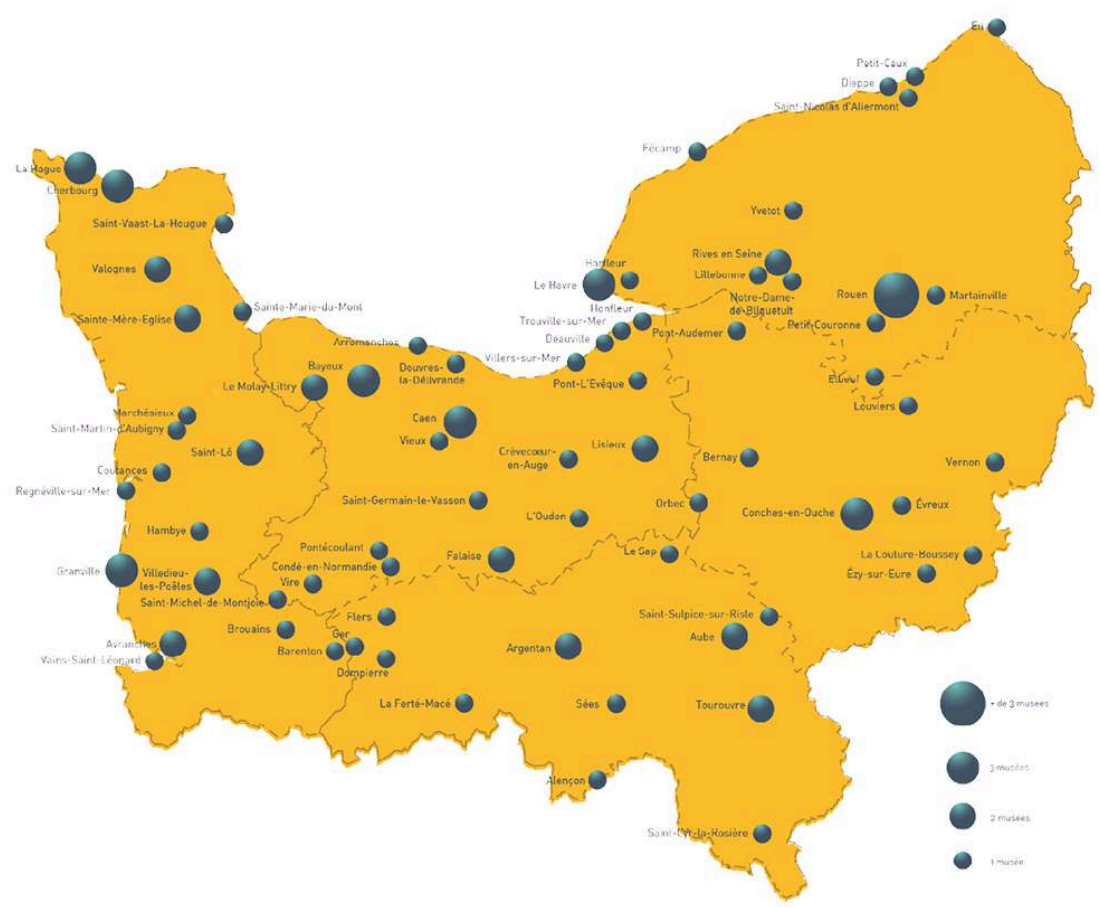


1 Le Réseau des musées de Normandie permet aux professionnels de près d'une centaine d'institutions réparties sur l'ensemble du territoire régional de se connaitre et de coopérer pour la conservation et la valorisation de leurs collections. Il est animé par l'un des pôles de la Fabrique de patrimoines en Normandie, un établissement public de coopération culturelle (EPCC $)^{1}$ financé par le Conseil régional de Normandie et la Direction régionale des Affaires culturelles (Drac). Le Réseau rassemble aujourd'hui plus de cent musées, que l'on peut qualifier pour la plupart de «petits musées » au regard de leurs moyens humains, traitant de thématiques variées : arts, archéologie, ethnologie régionale et extra-européenne, histoire naturelle, techniques, industrie...

Créé en 2003 en Basse-Normandie, le Réseau devient progressivement " normand » depuis le $1^{\mathrm{er}}$ janvier 2016, date d'entrée en vigueur de la nouvelle délimitation des régions ${ }^{2}$. Cette extension à deux nouveaux départements vient enrichir les possibilités de coopération, tout en posant un véritable défi opérationnel d'adaptation des méthodes et outils. Comment, du point de vue de l'animation de ce réseau, maintenir une continuité tout en intégrant un nouveau territoire? Quels sont les effets de cette extension sur les orientations stratégiques et sur les actions du réseau?

\section{Une culture de réseau contrastée}

3 Dès les années 1990, plusieurs musées bas-normands ont manifesté une volonté de travailler conjointement. Le Centre régional de culture ethnologique et technique de Basse-Normandie (Crécet), association loi 1901, qui travaillait en relation étroite avec de nombreux musées de société normands, fut moteur d'une réflexion sur la création d'un réseau et la porta auprès de ses tutelles. C'est dans le cadre d'un contrat de plan que l'État et la Région Basse-Normandie construisirent une politique commune de création d'un tel réseau. Suite à une étude réalisée par un cabinet privé, le Réseau fut créé en 2003 et rassemblait alors une trentaine de musées de société, c'est-à-dire traitant d'ethnographie locale; sa gestion et son animation furent confiées au Crécet. En 2012, le nombre d'adhérents était passé sans difficulté à soixante. L'appellation « de société » est alors tombée car la nature des musées adhérents s'était petit à petit élargie, avec de nombreux musées pluri-thématiques inclassables. En 2015, le Crécet devient La Fabrique de patrimoines en Normandie, suite à une fusion avec deux autres associations patrimoniales.

4 En Haute-Normandie, l'histoire est bien différente : la mise en réseau des musées n'a pas été portée par une politique régionale, mais directement par les professionnels des musées, rassemblés depuis les années 1980 dans une section fédérée de l'Association Générale des Conservateurs des Collections Publiques de France (AGCCPF). Cette association de professionnels avait mis en place avec succès un site web commun et des groupes de travail, mais demeurait fragile en raison du manque de moyens, notamment humains. Les bénévoles de cette association ont donc été très enthousiastes à l'idée que leurs musées rejoignent le Réseau et puissent s'appuyer sur une animation confiée à une personne dédiée. Le dialogue entre la Fabrique de patrimoines en Normandie et l'AGCCPF, qui existait déjà avant la fusion des régions, s'est établi en toute fluidité pour bien définir les périmètres d'action de chacun ${ }^{3}$. Les musées, incités par l'association et par la Drac, ont rejoint massivement le Réseau entre 2016 et 2018, avec 30 adhésions en 2 ans. 
Le travail de mise en réseau, dont l'objectif est d'installer une confiance mutuelle et la volonté de travailler réellement ensemble, au-delà des apparences, ne peuvent porter leurs fruits que sur un temps long. Le bouleversement des frontières régionales est venu bousculer cette temporalité, créant un déséquilibre entre les départements de l'Ouest, familiers du Réseau, et ceux de l'Est. Comment fédérer harmonieusement les convaincus de l'utilité d'un réseau régional et ceux qui restent à convaincre?

Les rencontres entre membres du Réseau sont fondées sur le partage d'expérience et les échanges.

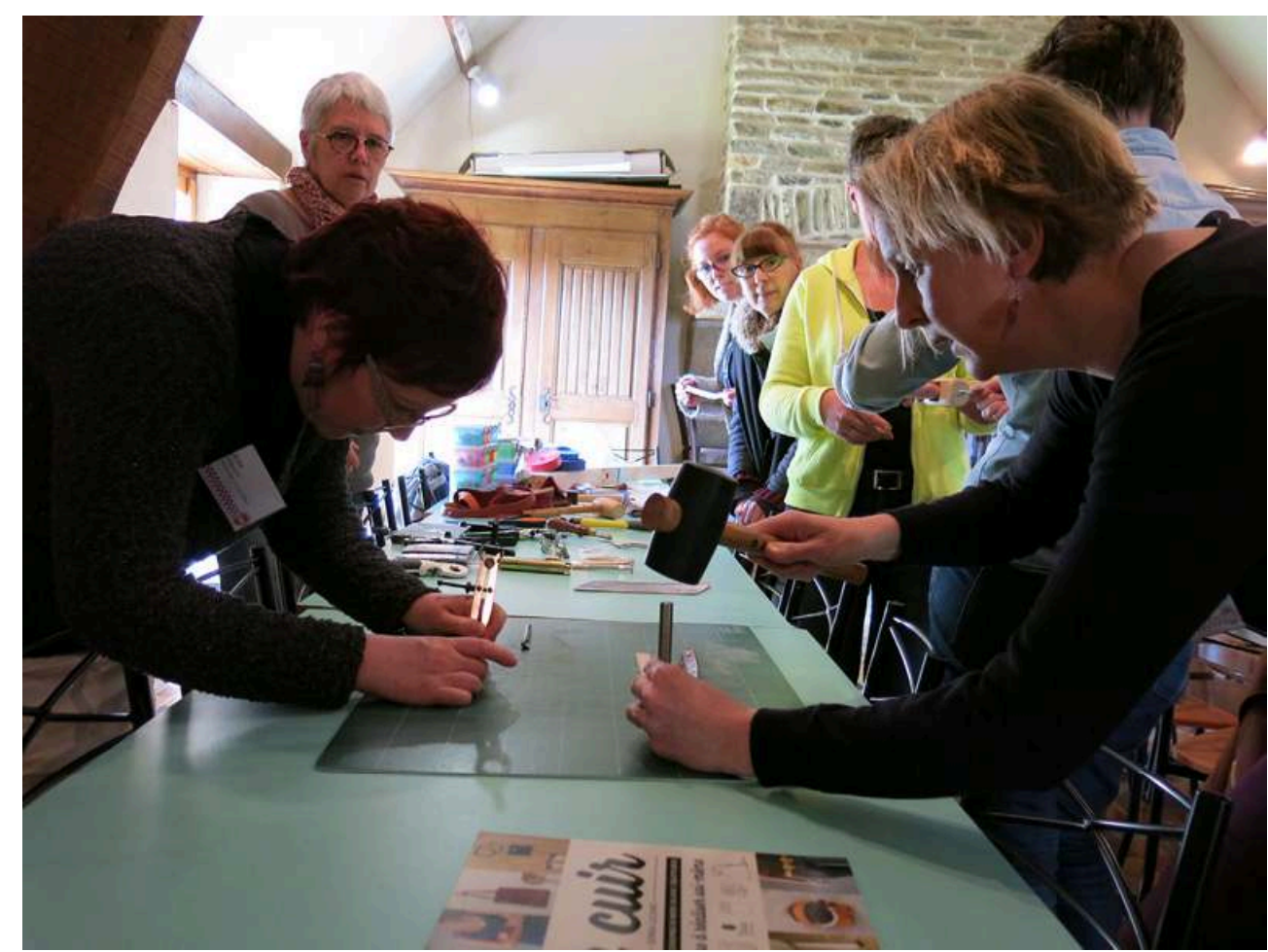

(c) Margot Frénéa

\section{Un réseau ouvert et bien structuré}

Depuis 2017, le pôle Réseau est constitué de trois personnes : une coordinatrice, une administratrice de base de données des collections, une chargée de communication et de médiation. Il est soumis à une double gouvernance : l'une « ascendante » depuis les membres, via le Comité d'orientation; l'autre " descendante » depuis les tutelles, qui arbitrent les budgets et leurs usages en Conseil d'administration de la Fabrique.

Le Réseau des musées de Normandie propose, depuis sa création, une adhésion gratuite. Pour devenir membre, une structure doit répondre à la définition du musée de l'Icom (Conseil international des musées): un filtre important est celui du «but non lucratif », qui permet néanmoins d'accepter des statuts privés (associations, fondations), à condition que les collections appartiennent au domaine public. Le Réseau réunit la plupart des Musées de France de la région, mais pas seulement: les petits musées associatifs fonctionnant sur le bénévolat sont également les bienvenus. 
8 Lorsqu'un musée fait une demande d'adhésion, sa candidature est traitée par le Comité d'orientation du Réseau. En 2016, face au nombre important de musées candidats dans l'Eure et la Seine-Maritime, il a été décidé d'accepter automatiquement les Musées de France, partant du principe que s'ils répondaient à l'appellation nationale, ils correspondaient au moins à la définition de l'Icom.

Ce Comité d'orientation est composé d'un représentant de chaque tutelle (Drac et Région) et de deux collèges : cinq personnes représentant les élus des collectivités porteuses de musées et cinq personnes représentant les professionnels des musées membres. Ils sont élus pour deux ans par les musées membres lors de l'Assemblée plénière annuelle. Ce comité, qui se réunit deux à trois fois par an, est consulté pour orienter les actions menées par l'équipe de la Fabrique et arbitre les nouvelles adhésions. En novembre 2018, parmi onze candidats au Comité d'orientation, un seul venait d'un musée du territoire de l'ancienne Haute-Normandie et a été largement élu. Le processus d'appropriation des instances du Réseau par les professionnels de l'ancienne Haute-Normandie va sans doute prendre du temps, mais les premières pierres sont d'ores et déjà posées.

\section{Équité et collaboration}

La mise en réseau repose sur des principes qui ne sont pas nécessairement énoncés dans les statuts, mais qui teintent profondément les discours sur le Réseau et orientent le type d'actions mises en place.

11 Premier principe : équité et horizontalité. Le territoire normand réunit à la fois des " poids lourds » nationaux par leur fréquentation et des musées plus modestes situés dans des zones rurales moins touristiques. Or le Réseau tend à valoriser les bonnes pratiques dans tous les musées, quelles que soient leur taille et leur nature : les grands musées ont (aussi) beaucoup à apprendre des plus petits !

Deuxième principe : œuvrer pour l'amélioration des pratiques dans les musées. Pour les musées associatifs, l'objectif n'est pas nécessairement la professionnalisation, mais la montée en qualité, y compris pour les bénévoles. Le Réseau est ouvert aux musées qui n'ont pas l'appellation Musée de France : le travail d'accompagnement, notamment sur les collections, participe à la protection des œuvres dont le statut n'est pas sécurisé. La relation de proximité avec les acteurs des musées est de plus en plus difficile à maintenir au regard du nombre d'acteurs concernés (environ 500 professionnels et bénévoles actifs).

Un troisième principe, qui peut sembler évident, est la dimension collaborative et inclusive du Réseau qui concerne tout le personnel des musées. Les actions sont donc diversifiées afin de faciliter les échanges entre personnels de différentes institutions pour tous les métiers, de l'accueil-surveillance à la conservation scientifique, en passant par la médiation ou la gestion de boutique. Les membres du Réseau sont régulièrement consultés et impliqués dans les choix d'orientation des actions menées, via des votes ou questionnaires en ligne par exemple.

14 Un autre principe tend à s'imposer suite à l'élargissement du Réseau : le rôle de l'équipe du pôle Réseau est de créer un environnement favorable au développement de la coopération. La proportion entre les moyens du pôle et le nombre de musées (3 postes pour 100 musées) accentue de plus en plus ce positionnement. L'équipe crée et 
administre des outils communs afin de faciliter la coopération : à ce jour, une base de données et un logiciel de gestion des collections mutualisés, un portail des collections régionales et un site web collectif. Pour l'avenir, une plateforme d'échange est à l'étude, regroupant des fonctionnalités d'annuaire, de discussion, de partage de veille et de ressources.

Les cafés muséos proposent des formats de rencontre divers : ici, un atelier pratique d'échange de savoir-faire entre médiateurs et médiatrices.

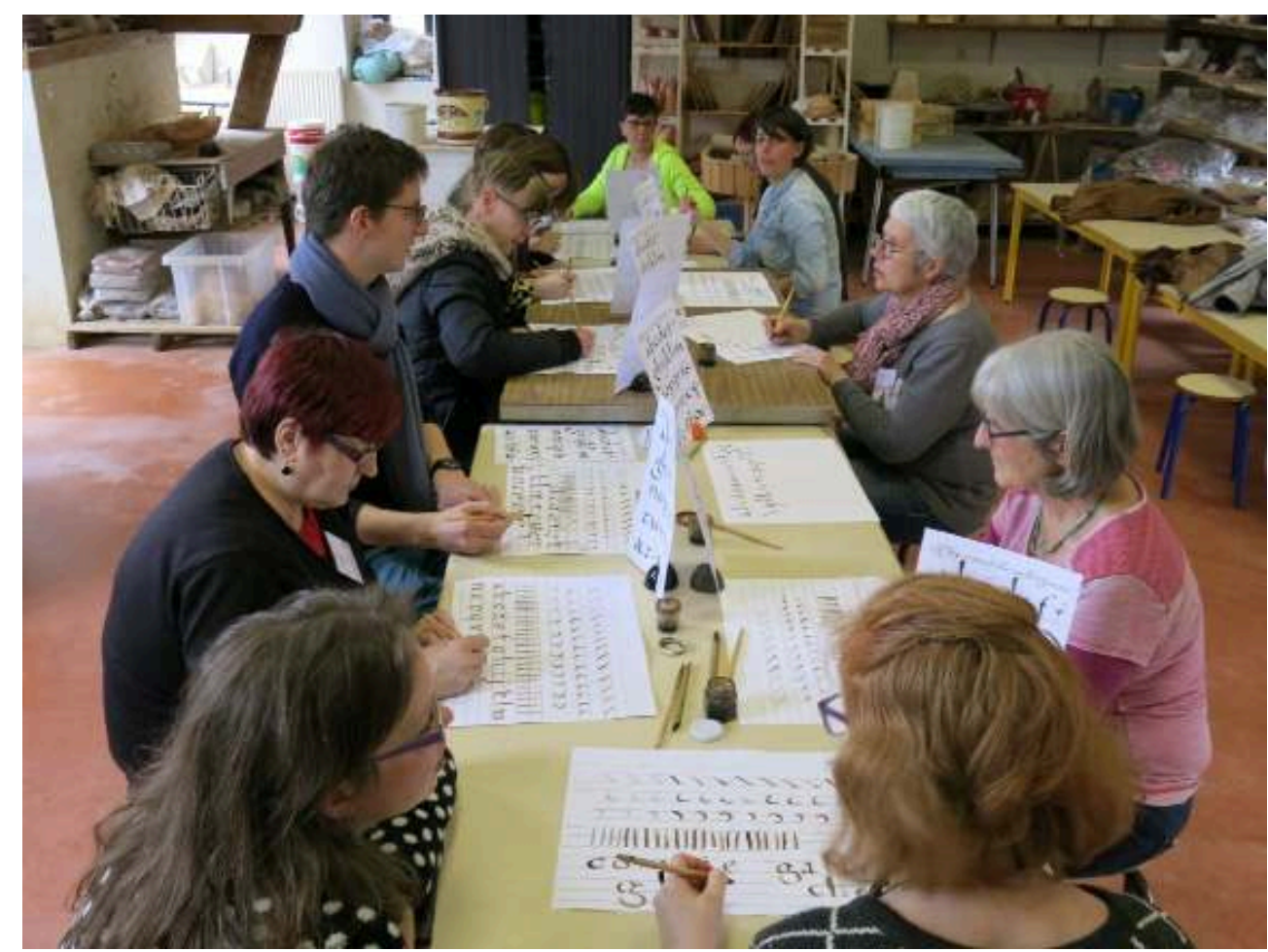

(C) Margot Frénéa 
Les temps d'échange informels permettentaux membres du Réseau de mieux se connaitre, condition nécessaire à toute coopération.

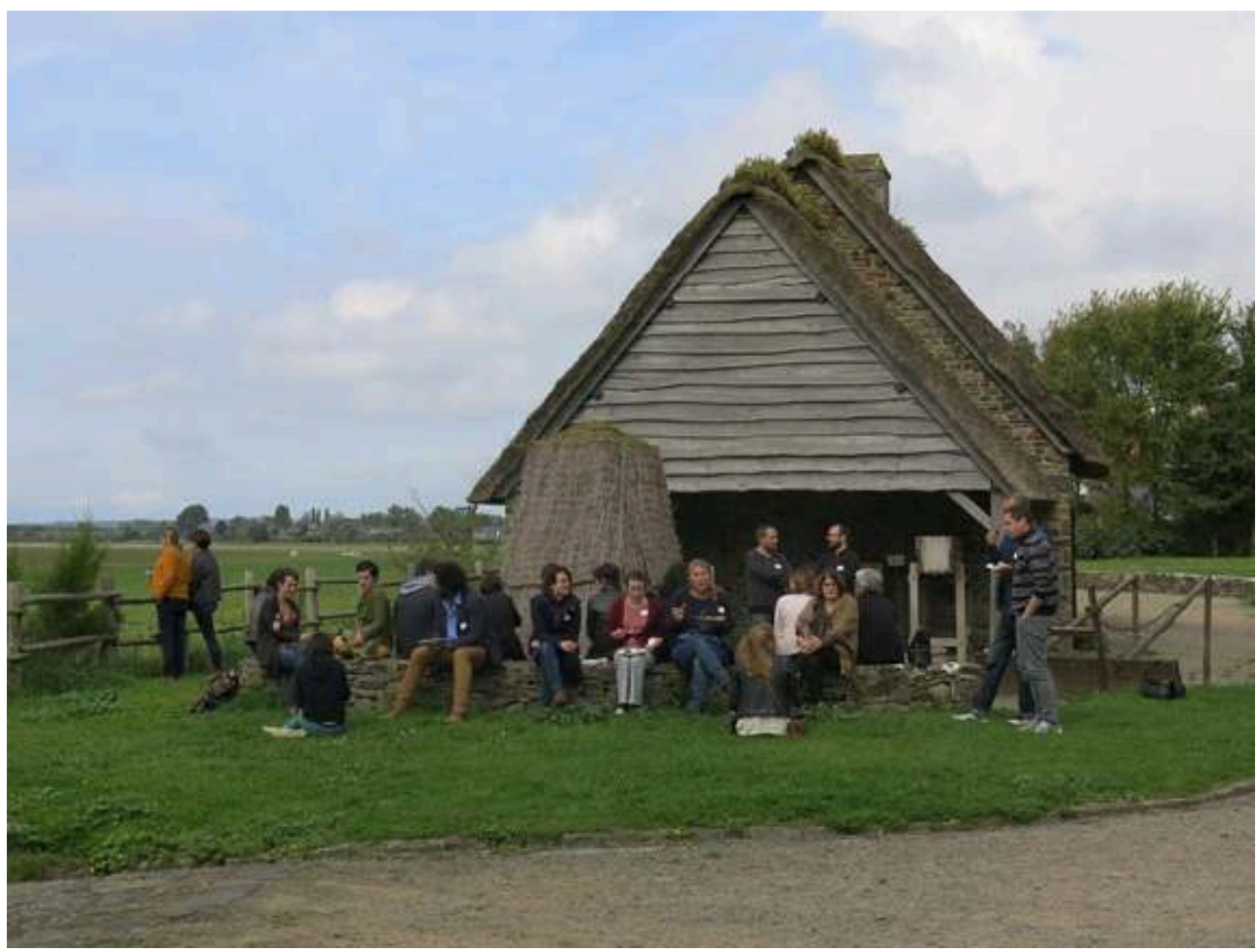

(c) Margot Frénéa

\section{Les actions du Réseau}

15 Afin de promouvoir les musées membres, le Réseau présente leurs activités et leurs actualités sur un site web qui a été refondu et adapté au nouveau territoire régional (sorti en avril 2017). L'équipe du pôle Réseau relaie également les activités des membres sur les réseaux sociaux numériques (Facebook et Twitter). L'augmentation du nombre de musées entraîne un accroissement du travail de veille et de relais, tout en accentuant la difficulté de rester équitable.

Afin que le Réseau soit concret pour les acteurs des musées, les rencontres réelles sont indispensables. C'est pourquoi 4 à 5 cafés muséos sont organisés chaque année, ainsi qu'une rencontre "Musées et numérique »" Ces réunions sont parfois vécues par les personnels isolés comme des "bouffées d'oxygène ». Les cafés muséos sont accueillis par les musées membres et réunissent entre 30 et 60 personnes autour de thématiques variées. La nouvelle échelle de territoire renforce les effets de polarité géographique et oblige à varier les lieux avec un soin particulier pour un égal accès à ces rencontres.

Dans l'objectif d'améliorer les pratiques des professionnels des musées membres, 5 à 7 formations sont organisées chaque année depuis 2017, pour certaines en partenariat avec le CNFPT (Centre national de la Fonction publique territoriale) et l'Ocim.

Une action fondamentale pour la mise en réseau consiste en la transmission d'informations entre les membres du Réseau. Le répertoire de contacts des personnels des musées a considérablement augmenté, naturellement suite à la hausse du nombre d'établissements, mais aussi grâce au Muséopass (une carte d'accès gratuit aux musées 
réservée aux personnels des musées membres) dont les demandes permettent de maintenir à jour ce fichier. En outre, une Mus'letter mensuelle est envoyée à tous, et complétée ponctuellement par des messages d'informations choisies sur demande des membres (proposition de dons, événement scientifique, recherche de matériel muséographique en prêt, recherche de collections en vue d'une exposition temporaire...).

Depuis quelques années, la Fabrique de patrimoines a souhaité mettre en place un Observatoire des musées, réalisé par le pôle Réseau et le pôle Conservationrestauration. L'objectif est de récolter des données fiables et complètes, et de produire des statistiques sur les musées de la Région, d'une part pour alimenter les pratiques des personnels des musées membres, d'autre part pour améliorer la connaissance des musées de la région. Le premier Observatoire a porté sur l'année 2015, puis a été reconduit pour l'année 2016. Mais le territoire de référence a entre temps changé, ce qui complique la production des résultats au regard de la masse de données à traiter. Cette expérimentation est donc appelée à évoluer pour conserver toute sa pertinence.

Le pôle Réseau a également un rôle de conseil et d'accompagnement : en développant une relation de confiance, en dehors de tout contrôle règlementaire et de toute attribution de subvention, l'équipe apparaît souvent comme un interlocuteur privilégié. Ce dernier se fait alors relais vers des ressources méconnues ou renvoie vers des contacts précis pouvant témoigner d'une expérience inspirante.

La mise en réseau repose sur la participation des membres à travers des outils collaboratifs.

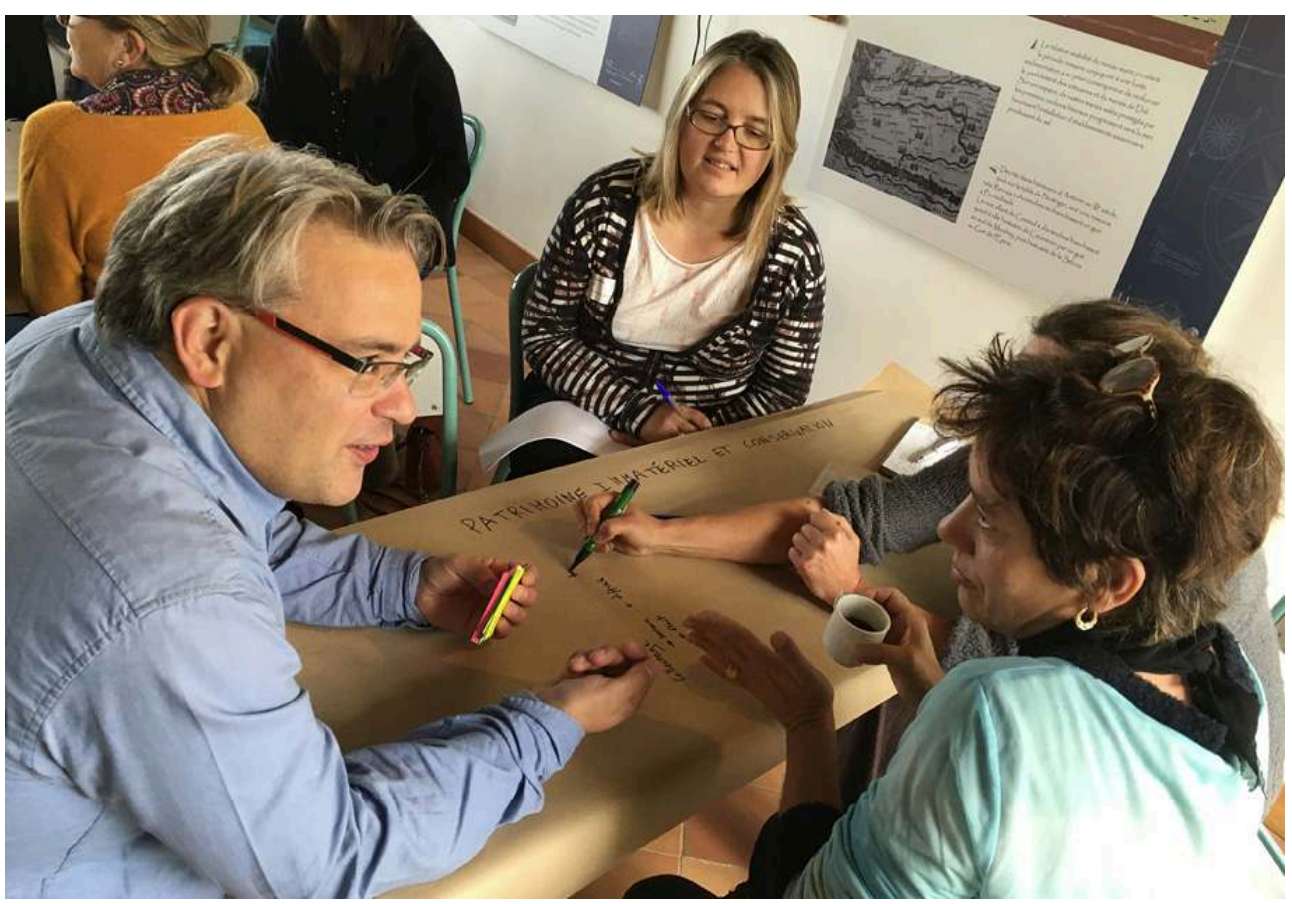

(c) Margot Frénéa 


\section{Les outils en faveur de la conservation, l'étude et la diffusion des œuvres}

L'un des principaux enjeux du Réseau depuis sa création est la connaissance et la sauvegarde du patrimoine muséographique normand. Pour répondre à cet objectif, le numérique est rapidement apparu comme le seul outil permettant d'assurer capitalisation, centralisation et sauvegarde des savoirs. Parmi les outils administrés par le Réseau, l'exemple le plus poussé de mutualisation et de coopération entre les musées est sans nul doute la base de données des collections qui permet, entre autres, la mise en ligne des données documentaires. La sauvegarde des collections reste, quant à elle, l'apanage des musées, le pôle Réseau n'intervenant à ce sujet que par le biais de conseils.

\section{Le Réseau en quelques chiffres \\ 105 musées adhérents en 2018 \\ dont 69 Musées de France \\ $50 \%$ des musées membres ont moins de 3 personnes salariées \\ $75 \%$ des musées membres ont moins de 5 personnes salariées \\ (données issues de l'Observatoire des musées 2016)}

Chaque formation (comme ici celle consacrée aux maquettes) est conçue sur mesure à partir des demandes des professionnels et de la connaissance de leurs problématiques.

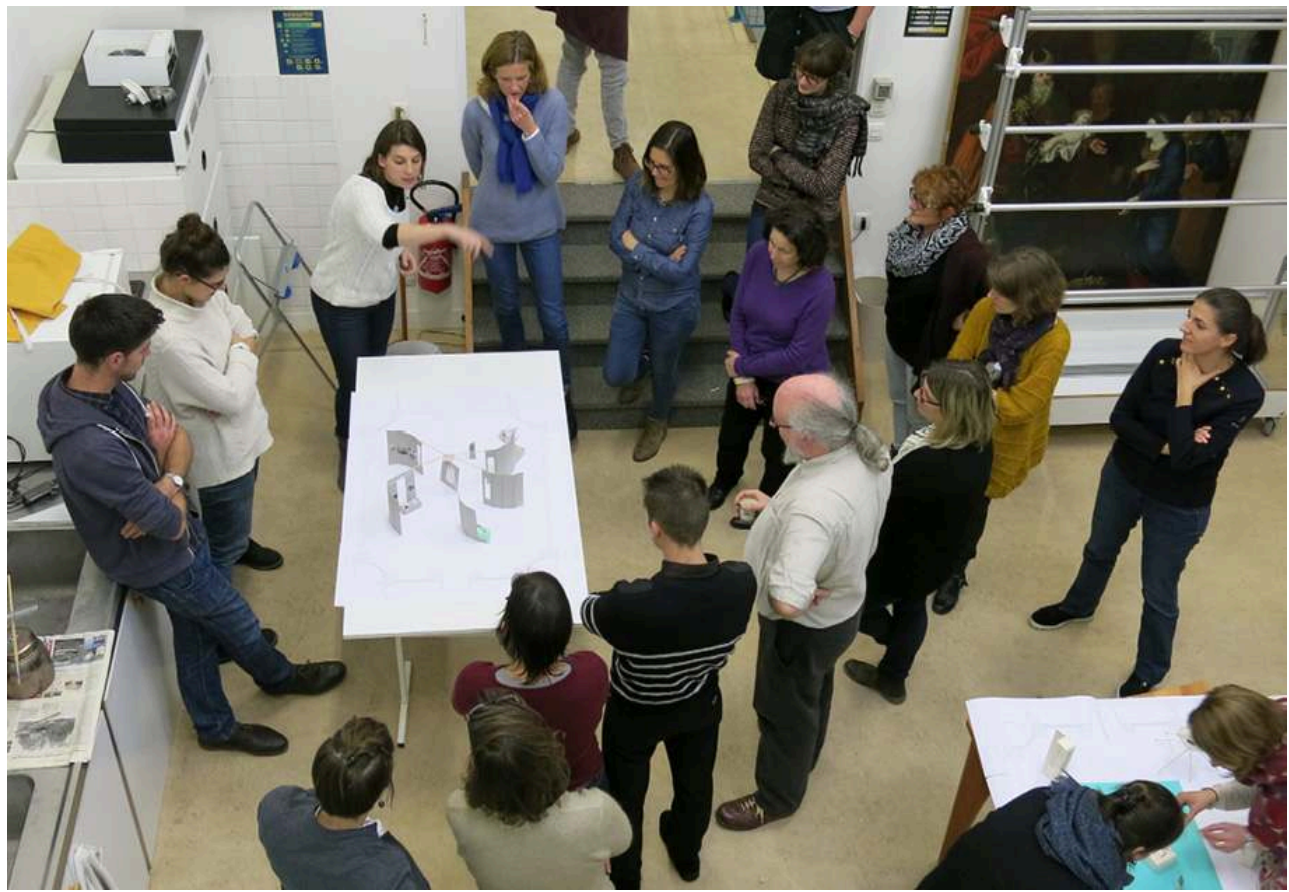

(c) Margot Frénéa 


\section{La base de données mutualisée des collections} d'administration de l'outil consiste à assurer la cohérence du système notamment grâce à la mise en place de protocoles et de règles communes. Cette recherche d'harmonisation est complexe et chronophage au regard du nombre d'institutions et d'utilisateurs. En effet, les usages et compréhensions du système diffèrent potentiellement pour chaque établissement. Il est par ailleurs impossible de vérifier chaque nouvelle saisie, ce qui représente un risque de baisse de qualité des données présentes. À l'inverse, il apparaît primordial de laisser une marge de manœuvre aux professionnels dans la gestion de leurs collections. Tout l'enjeu est donc de trouver l'équilibre entre procédure commune et charte de saisie spécifique, entre respect de l'existant et participation à l'évolution et au renforcement de l'outil partagé des utilisateurs.

Pour favoriser l'appropriation de l'outil par l'ensemble des usagers - que chacun puisse devenir acteur et contribuer à son bon fonctionnement - un club utilisateurs a vu le jour en 2017. Il s'agit d'encourager les échanges et les retours d'expérience autour de la base de données, ainsi que la mise en commun des bonnes pratiques. Les retours des 
utilisateurs jouent un rôle indéniable dans l'évolution et le paramétrage de l'application. La co-construction de l'outil fonctionne selon une démarche ascendante : partir des besoins spécifiques exprimés par l'échelon local, le musée, en favorisant les échanges et en maintenant une coordination assurée par l'échelon global, le Réseau. Ainsi, le travail d'administration comprend également cette approche collaborative avec le suivi des améliorations apportées à l'application qui ne cesse de s'adapter aux besoins et à l'évolution des métiers. Il est en effet indispensable de disposer d'un outil non figé, évolutif, adaptable, et de bénéficier d'un dialogue ouvert et maintenu avec la société éditrice.

Le site web du Réseau des musées propose à la fois un agenda des musées et des ressources sur les publications des musées, les expositions itinérantes...

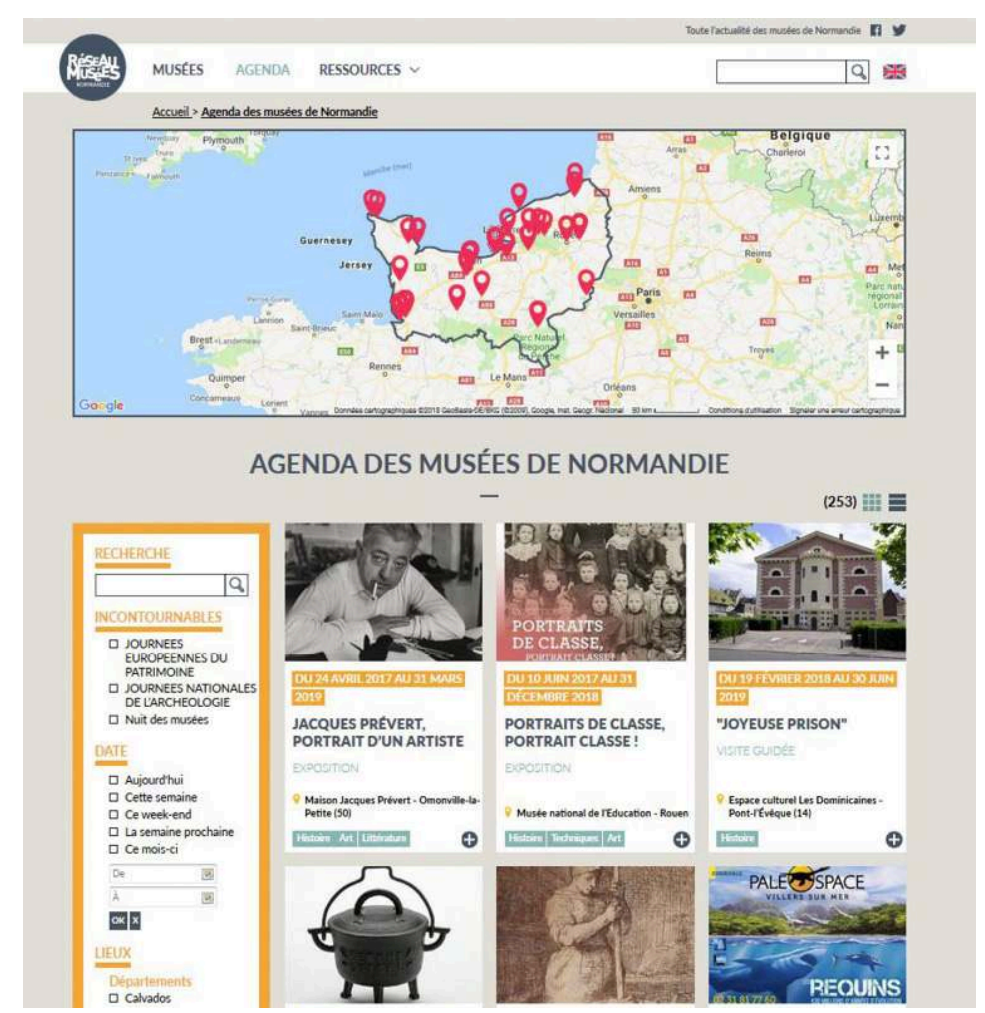

(c) Site web conçu par Louise Marnai (graphisme) et Nicolas Villeroy (développement)

\section{Un nouveau Portail des collections}

Un Opac Web ${ }^{6}$, nommé Muséobase, a été installé en 2012 afin de révéler les collections aux publics en rendant accessibles les données communicables sur les œuvres. Ainsi, chaque musée est libre de publier les notices de ses collections sur un site web offrant une vision transversale du patrimoine régional. Il s'agit aussi de valoriser le travail scientifique et documentaire réalisé sur les objets par les professionnels, de créer une proximité et d'encourager une appropriation des objets et des œuvres par les publics. En 2015, un dispositif d'indexation participatif a été mis en place permettant aux internautes de laisser des commentaires sur les notices. Partant du constat d'un manque d'information et de documentation sur un certain nombre d'objets, il est apparu important de prendre en considération la connaissance, mais aussi la mémoire 
de la population, notamment locale. De plus, l'évolution du numérique incite à revoir les relations avec les publics, à travailler de plus en plus selon des méthodes relationnelles laissant une part importante à la co-production, notamment en cherchant à fidéliser les implications du public.

Plutôt qu'un simple catalogue des collections, la Fabrique de patrimoines souhaite désormais proposer un véritable portail qui offrira de nouveaux services et des fonctionnalités supplémentaires (développement de l'aspect contributif sans oublier le côté ludique), et, à terme, d'autres ressources documentaires. Ce projet de refonte, débuté en janvier 2018 et dont le lancement est prévu pour 2019, répond à plusieurs besoins :

29 - donner une vision plus globale du patrimoine muséographique conservé et aider à sa découverte (navigation plus aisée et suggestive) ;

30 - proposer un outil innovant et dynamique qui bénéficiera d'une animation accrue ;

31 - accompagner les projets collaboratifs grâce à la mise en place de nouvelles formes de médiations autour des objets (galeries thématiques, jeux, réutilisations...).

Toutefois, en tout premier lieu, il s'agit de s'adapter au territoire redéfini en prenant en compte la diversité des usages. Pour ce faire, le nouveau portail ne sera plus dépendant de la base de données mutualisée, mais permettra de récupérer des données issues de plusieurs outils documentaires.

33 Une fois les aspects techniques pris en compte, il sera nécessaire que les professionnels des musées modifient leurs habitudes de saisie avec, à l'esprit, un objectif de médiation renouvelé. En effet, de nouveaux protocoles sont à prévoir permettant de passer d'une logique de numérisation des données sur les collections pour une gestion administrative à une logique de diffusion et d'ouverture pour les publics. Cela suppose un investissement et un engagement important des professionnels qui devront modifier certaines notices (éviter les saisies de type catalogage pour rédiger des phrases complètes, se contraindre à ne pas employer des champs documentaires pour des notes personnelles ou de travail, ne pas utiliser d'abréviations...). Ainsi, seule l'implication de tous les acteurs - au quotidien et sur le long terme - garantira la qualité des notices d'œuvres diffusées en ligne.

34 Enfin, la mise en place de partenariats avec le milieu de l'enseignement est au cœur des enjeux du portail. Celui-ci permettra de proposer aux enseignants un outil-ressource avec la possibilité d'inscrire des projets dans le parcours d'éducation artistique et culturelle des élèves. L'objectif est ici double : poursuivre la diffusion des collections auprès des jeunes publics et disposer d'une communauté relais dans une démarche de coopération avec les musées. 


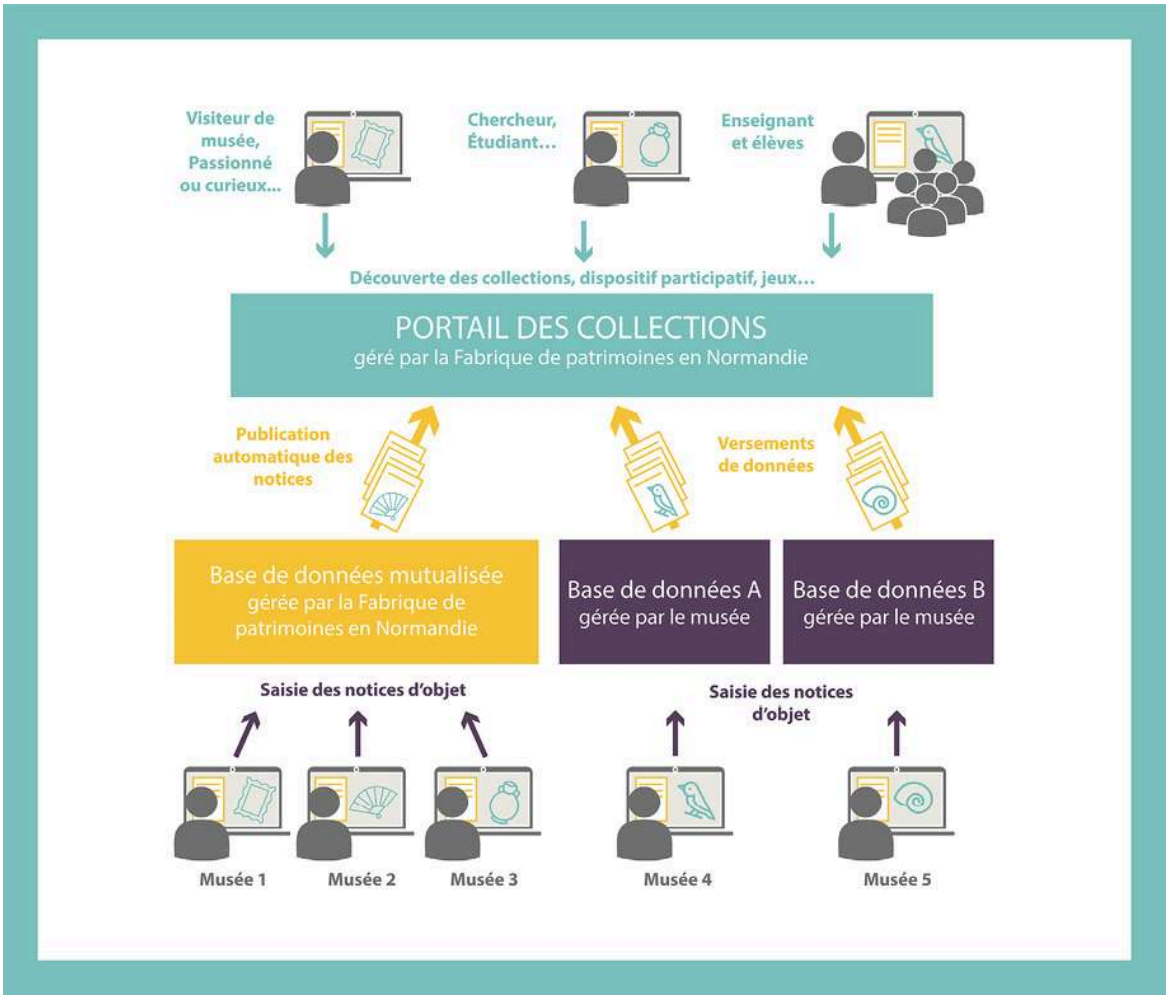

(c) La Fabrique des patrimoines

\section{Conclusion}

L'extension du territoire de référence du Réseau des musées de Normandie a pour conséquence une modification des méthodes d'animation, conduisant le pôle Réseau à adapter ses actions, d'une part au nombre accru de membres et aux distances géographiques, d'autre part à des cultures professionnelles et à des historiques différents. La participation aux actions du Réseau reposant sur la volonté de chacun, le défi de l'animation de réseau consiste donc, avec encore plus d'acuité, à susciter l'implication des musées par la qualité des actions proposées.

Frénéa, M. et Schmit, P. La mise en réseau : de nouvelles perspectives pour les musées? in Amsellem E. et Limousin I. Le Musée, demain. Actes du colloque de Cerisy de juin 2014, Paris : L'Harmattan, 2017, pp. 173-181.

Schmit, P. et Lassalle-Astis, L. Le Réseau des musées de société en BasseNormandie, Musées et collections publiques de France, n²41, Tome 1, 2004, pp. 86-89. Centre régional de culture ethnologique et technique. Réseau des musées de société en Basse-Normandie. Rapport de projet. Avril 2003.

Site web du Réseau des musées de Normandie : www.musees-normandie.fr Site web des collections en ligne du Réseau des musées de Normandie : www.museobase.fr 


\section{NOTES}

1. Cet EPCC a été créé au $1^{\mathrm{er}}$ janvier 2015 à partir de la fusion des associations suivantes : le Crécet, l'Arcis et Normandie patrimoine.

2. La Basse-Normandie comprenait trois départements (le Calvados, la Manche et l'Orne) et la Haute-Normandie deux départements (l'Eure et la Seine-Maritime), désormais rassemblés pour former la Normandie.

3. Dans les grandes lignes, l'association se concentre sur la défense de la profession tandis que le Réseau met en place coopération et mutualisation.

4. Les cafés muséos sont des journées de partage d'expérience et de discussion sur des thématiques muséales variées, choisies par vote par les membres du Réseau. Exemples: les boutiques de musée, mécénat et financement participatif, musées sur le web, les abords des musées, partage de savoir-faire en médiation, etc. Les rencontres « Musées et Numérique » sont organisées en partenariat avec le pôle TES.

5. Full web qualifie une application accessible en ligne, grâce à une simple connexion à internet via un navigateur web, sans installation d'une application sur poste.

6. Opac Web, acronyme pour " Online Public Access Catalog ", désigne une interface en ligne de consultation d'une base de données documentaire.

\section{RÉSUMÉS}

Favoriser la connaissance mutuelle des acteurs du territoire et le partage d'informations, d'expériences et de savoir-faire, promouvoir les musées membres et susciter des projets collectifs : les auteures montrent comment la mise en place d'outils numériques mutualisés - site internet, base de données et portail des collections - et l'accompagnement proposé permettent au réseau de répondre à ces missions.

\section{INDEX}

Mots-clés : coopération, réseau, développement territoriale

\section{AUTEURS}

\section{ÉLISE FIN-OSINSKI}

Administratrice de la base de données des collections du Réseau des musées de Normandie elise.fin-osinski@lafabriquedepatrimoines.fr

\section{MARGOT FRÉNÉA}

Coordinatrice du Réseau des musées de Normandie

margot.frenea@lafabriquedepatrimoines.fr 